\title{
Celiac Disease and Non-Celiac Gluten Sensitivity; Evidences and Differences
}

\author{
Rostami Nejad, Mohammad* \\ Celiac disease department, Gastroenterology and Liver diseases Research Center, Shahid Beheshti University of Medical Sciences,
} Tehran, Iran

But in children younger than 2 years, the IgA TTG test

\section{Introduction}

There are too many papers published regarding the definition, diagnosis and treatment of celiac disease (CD). All we know that CD is a major health care issue affecting people in any ages, and increased wheat consumption may have had an effect on CD prevalence, which was reported worldwide prevalence of approximately 1\% [1].

Most of the celiac patients report long gastrointestinal symptoms such as abdominal pain, tenderness, or irregular bowel habits like constipation or diarrhea or alternating bowel movements. Very often they consult a number of physicians, seeking to reach a CD diagnosis but they are considered to be simply suffering from irritable bowel syndrome (IBS) [2].

According to ACG clinical guidelines, in individuals over the age of 2 years, anti-tissue transglutaminase (TTG) immunoglobulin A (IgA) antibody is strongly recommended and preferred for celiac disease diagnosis. should be used combined with DGP (IgA and IgG) [3].

Currently, the only safe and effective treatment is a strict gluten-free diet (GFD) accompany with nutritional support, which improves the health and quality of life in the vast majority of patients [4]. There is no doubt that GFD so effective in symptomatic CD patients, and reduce the risk of malignancies such as osteoporosis, refractory CD and small intestinal lymphoma [5].

On the other hands, a consistent percentage of the general population consider themselves to be suffering from troubles caused by wheat and/or gluten ingestion, even if they do not have celiac disease or wheat allergy (WA), as they test negative both for CD serology and histopathology and for IgE-mediated assays. Most patients report both gastrointestinal and non-gastrointestinal symptoms, all agreeing, however, about the reduction of symptoms on a gluten-free diet. This clinical condition has been recently named Non-Celiac Gluten Sensitivity (NCGS) [6]. We talk about NCGS when CD serology, duodenal histology and IgE-based assays are negative (Table 1).

Table 1. Laboratory and clinical criteria for distinguish between celiac disease and non-celiac gluten sensitivity

\begin{tabular}{|l|c|c|}
\hline \multicolumn{1}{|c|}{ Variables } & Celiac disease & Non Celiac Gluten sensitivity \\
\hline Celiac disease serology: & & negative \\
- anti-tissue transglutaminase & positive & negative \\
- anti-endomysial antibodies & positive & negative \\
- deaminated gliadin peptide antibodies & positive & positive (50\% of the cases) \\
- anti-gliadins antibodies & positive & (Marsh 0-II) \\
\hline Histology abnormalities & (Marsh I-IIIc) & positive (50\% of the cases) \\
\hline HLA DQ2/DQ8 haplotypes & present & negative \\
\hline IgE-based assays & negative & Intestinal and extra intestinal symptoms \\
\hline Clinical features & gluten-free diet & gluten-free/wheat-free diet \\
\hline Treatment & Intestinal and extra intestinal symptoms & innate immune response \\
\hline Immunology response & innate and adaptive immune response & \\
\hline
\end{tabular}

As presented in Table 1, NCGS can be associated with mild abnormal histology and could not detectable by gastroendoscopy. Such lesions do not change to partial or total villous atrophy during contact by gluten but will be improved after strict GFD.

These patients do not believe that gluten is considered to be the cause of their symptoms; therefore they continue to consume gluten-containing foods. As a result, they were left in a no man's land, unrecognized by either allergists or gastroenterologists. Due to poor physician's awareness of this disease, NCGS patients were commonly referred to psychiatrists because they were believed to have an underlying mental illness like IBS $[7,8,9,10]$.

However, the pathogenesis of NCGS is still unclear, but improvement in these patients with gluten withdrawal is associated with HLA-DQ2/DQ8 positivity. HLA-DQ2/8 positive patients had faster small bowel and/or colonic transit compared with HLA-DQ2/8 negative patients [11].

Although CD and NCGS seem to be two different conditions, it has been reported that 10 of 78 (12.8\%) patients with NCGS were first-degree relatives of CD patients [9]. In this regard, we suggest the usefulness of the HLA haplotype determination to search for the DQ2 or DQ8 forming alleles. In fact, due to the high negative predictive value of the genetic assay, in the patients who will result negatives for DQ2 and DQ8 haplotype, the CD diagnosis can be excluded.

Furthermore, it is known that local in-rectum gluten instillation can be a useful test to identify mucosal evidence of NCGS at an early stage in asymptomatic firstdegree relatives of $\mathrm{CD}$ patients $[12,13]$. The final diagnosis of NCGS is gluten challenging. Gluten 
withdrawal is associated with an impressive improvement or even the disappearance of IBS-like and extraintestinal symptoms, and reintroducing gluten causes symptom recurrence. Cereals, such as buckwheat, rice, corn and millet, and vegetables, are recommended as substitutes for gluten-containing products.

In conclusion, diagnosis NCGS should be confirmed by $\mathrm{CD}$ and wheat allergy exclusion, along with a personal history of food allergy in infancy, coexistent atopy, positivity for IgA AGA and duodenal and/or ileum-colon intraepithelial and lamina propria eosinophil counts. However, future studies should be performed to identify reliable biomarkers for NCGS diagnosis and to better define the different NCGS subgroups.

\section{References}

[1] Rostami Nejad M, Rostami K, Emami MH, Zali MR, Malekzadeh R. Epidemiology of Celiac disease in Iran; A Review. Middle East Journal of Digestive Diseases. 2011; 3(1): 74-77.

[2] Verdu EF, Armstrong D, Murray JA. Between celiac disease and irritable bowel syndrome: the "no man's land" of gluten sensitivity. Am J Gastroenterol 2009;104: 1587-1594.

[3] Rubio-Tapia A, Hill ID, Kelly CP, Calderwood AH, Murray JA. ACG Clinical Guidelines: Diagnosis and Management of Celiac Disease. AM J Gastrointerol 2013; 108: 656-76.

[4] Casellas F, Rodrigo L, Vivancos JL, Riestra S, Pantiga C, Baudet JS, et al. Factors that impact health-related quality of life in adults with celiac disease: a multicenter study. World J Gastroenterol 2008; 14: 46-52.

[5] Catassi C, Bearzi I, Holmes GK. Association of celiac disease and intestinal lymphomas and other cancers. Gastroenterology 2005; 128(4 suppl 1):S79-S86

[6] Aziz I, Sanders DS. Emerging concepts: from coeliac disease to non-coeliac gluten sensitivity. Proc Nutr Soc 2012; 71:576-580.

[7] Sapone A, Bai JC, Ciacci C, Dolinsek J, Green PH, Hadjivassiliou M, Kaukinen K, Rostami K, Sanders DS, Schumann M, Ullrich R, Villalta D, Volta U, Catassi C, Fasano A: Spectrum of glutenrelated disorders: consensus on new nomenclature and classification. BMC Med 2012; 10:13.

[8] Troncone R, Jabri B. Coeliac disease and gluten sensitivity. J Intern Med 2011;269: 582-90.

[9] Volta U, Tovoli F, Cicola R, Parisi C, Fabbri A, Piscaglia M, et al. Serological tests in gluten sensitivity (nonceliac gluten intolerance). J Clin Gastroenterol 2012; 46:680-685.

[10] Ludvigsson JF, Leffler DA, Bai JC, Biagi F, Fasano A, Green PH, et al. The Oslo definitions for coeliac disease and related terms. Gut 2013; 62:43-52.

[11] Vazquez-Roque MI, Camilleri M, Carlson P, McKinzie S, Murray JA, Brantner TL, Burton DD, Zinsmeister AR: HLA-DQ genotype is associated with accelerated small bowel transit in patients with diarrhea-predominant irritable bowel syndrome. Eur J Gastroenterol Hepatol 2011; 23:481-487.

[12] Dezi R, Niveloni S, Sugai E, Pedreira S, Smecuol E, Vazquez H, et al. Gluten sensitivity in the rectal mucosa of first-degree relatives of celiac disease patients. Am J Gastroenterol 1992; 92:1326-1330.

[13] Troncone R, Mazzarella G, Leone N, Mayer M, De Vincenzi M, Greco L, et al. Gliadin activates mucosal cell mediated immunity in cultured rectal mucosa from coeliac patients and a subset of their siblings. Gut 1998; 43:484-489. 